\title{
A V139M Mutation Also Causes the Reversible CNS Phenotype in CMTX
}

\author{
Michelle Halbrich, Jeffrey Barnes, Martin Bunge, Charuta Joshi
}

Can. J. Neurol. Sci. 2008; 35: 372-374

X-linked Charcot Marie Tooth disease (CMTX; OMIM \# $320800)$ is the second most common cause of inherited demyelinating neuropathy and is caused by mutations in the GJB1 ( also referred to as connexin 32) gene coding for the gap junction protein connexin $32(\mathrm{Cx} 32)$. $\mathrm{Cx} 32$ is expressed in the myelinating Schwann cells in the peripheral nervous system (PNS) and in oligodendrocytes in the central nervous system (CNS). Gap junctions help rapid transport of ions and small molecules across cell membranes. ${ }^{1}$ Various central nervous system manifestations (clinical and subclinical) in CMTX have been reported..$^{2-9}$ In the last ten years a distinct, reversible clinical syndrome associated with pyramidal and or bulbar weakness and concomitant transient magnetic resonance imaging (MRI) findings of diffuse, confluent white matter abnormalities has been increasingly reported..$^{10-13}$ All 11 patients reported in the published English literature since 1998, when the above syndrome was first described, have various mutations in the GJBl gene causing dysfunctional gap junctions. We report two brothers who presented with pyramidal weakness, dysarthria, ataxia and bulbar weakness a year apart predating their genomic CMTX diagnosis. Both boys and their mother were later confirmed to have a V139M mutation in the GJB1 gene. Computed tomography $(\mathrm{CT})$, magnetic resonance imaging and magnetic resonance spectroscopy (MRS) features of one of the brothers are described.

\section{Case 1}

A 13-year-old boy presented in February 2006 with episodes of transient neurological dysfunction on two consecutive days, two weeks after a concussion. He initially presented with left arm and leg weakness, dysarthria and a weak cough. He was thought to have developed an abnormal reaction to a cough syrup he had taken earlier in the day for cold symptoms. He was given intravenous benadryl and naloxone and, upon complete recovery, discharged without neurological consultation three hours later. He represented twelve hours later with bilateral ptosis, left facial weakness, dysarthria, a weak cough and generalised weakness with areflexia but with bilateral extensor plantar responses. This episode lasted eight hours and improved without intervention. Investigations included normal results for lumbar puncture, brain CT scan, CT angiogram, erythrocyte sedimentation rate, serum lactate, ammonia, serum amino acids, urine amino acids and organic acids, serum acetylcholine receptor antibody and stools for ova and parasites. Electromyography and Nerve Conduction Velocity (EMG/NCV) suggested a sensorimotor demyelinating and axonal neuropathy. Single fibre EMG was normal. Molecular testing for CMT type 1a was negative. The MRI was not performed acutely. During the following 12 months he developed classic migraines with good response to acetaminophen.

\section{CASe 2}

The 16-year old brother of the above patient, clinically diagnosed with CMT since age six years, presented to the emergency room in February 2007 with an acute onset of rightsided weakness and slurred speech two weeks after having recovered from pneumonia. Neurological examination revealed dysarthric speech, inappropriate laughter, right upper motor neuron facial weakness and moderate weakness of the right arm and leg. He had moderate bulbar weakness. Length dependent decrease in temperature and vibration sense and areflexia consistent with his clinically-diagnosed neuropathy was also noted.

His mother and multiple maternal relatives had a similar sensorimotor neuropathy. There was no family history of coagulopathies, vasculitides or congenital heart disease. He had residual mild hypophonia and bilateral intention tremor 21 hours after initial presentation. Six hours thereafter, he had acute onset of right arm weakness, dysarthria and progressive bulbar weakness. He remained alert and communicated with head nods. This episode lasted four hours and ended abruptly with emesis. In the month following discharge from hospital, he developed classic migraine.

Magnetic resonance imaging revealed symmetric nonenhancing prolonged signal intensity changes within the genu, body and splenium of corpus callosum and white matter in bilateral occipital lobe region on $\mathrm{T} 2$ weighted images and restricted diffusion in the same regions. Two single voxel MRS spectra obtained at an echo time (TE) of 144 did not reveal any lactate, glutamate, or lipid peaks. The MRS spectra also revealed

From the Department of Pediatrics (MH), Division of Pediatric Radiology (MB), Division of Pediatric Neurology (CJ), Children's Hospital Winnipeg; Section of Neurology (JB), Department of Internal Medicine, Health Sciences Centre, Winnipeg, Manitoba, Canada.

Received September 26, 2007. Final Revisions Submitted February 21, 2008 Reprint requests to: Charuta Joshi, 2506 JCP, Division of Pediatric Neurology, Children's Hospital of Iowa, 200 Hawkins Drive, UIHC, Iowa City, IA, 52242, USA. 


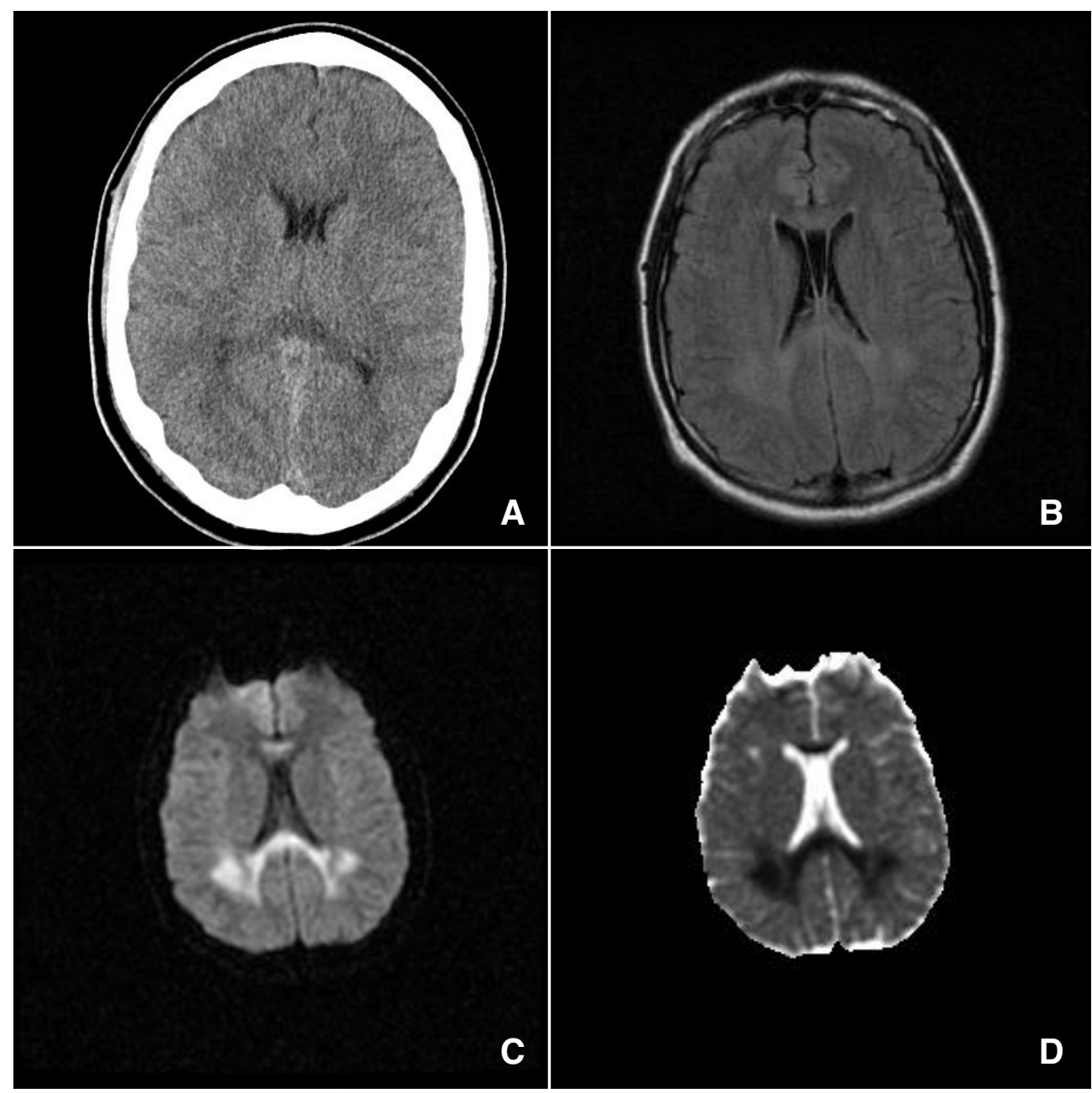

Figure. Initial non contrasted CT scan for Case 2 showing hypodense splenium, A) Axial Fluid Restriction Inversion Recovery (FLAIR) sequence B) and axial diffusion weighted images $C$ ) showing involvement of the corpus callosum and occipital white matter and apparent restricted diffusion coefficient map D) with similar findings as in $B$ ) and $C$ ).

a relative decrease in the creatine concentration. ACT angiogram (not shown) revealed smooth narrowing of the horizontal portion of the left anterior cerebral artery, thought to be a congenital variation. Other normal results included serum carnitine, ammonia, serum and urine amino and organic acids, long chain fatty acids, urine toxicology, blood counts, blood culture, electrolytes, ESR, C-reactive protein and serum immune electrophoresis. Cerebrospinal fluid analysis was normal. Hypercoagulable workup (factor 5 Leiden, Prothrombin G20210A mutation), vasculitis workup (serum homocysteine, antinuclear antibody and antiphospholipid antibody levels) and echocardiogram were normal. Mutation testing for Cerebral Autosomal Dominant Arteriopathy with Subcortical Infarcts and Leukoencephalopathy (CADASIL) was negative. Both patients and their mother subsequently tested positive for a mutation in the GJB1 predicted to cause a V139M variation in the Cx 32 protein. Repeat MRI and MRS after three months showed resolution of the white matter abnormalities seen earlier. The MRI and MRS for the younger brother (Case 1) at this time were normal.

\section{Discussion}

Central nervous system (CNS) involvement in CMTX is rare but well recognized. This has been previously reported in the form of abnormal evoked potentials, $, 2,3$ sensorineural deafness, ${ }^{5}$ extensor plantar responses ${ }^{4}$ and clinically silent scattered white matter hyperintensities on MRI ${ }^{8,9}$ CMTX has also been reported to present like MS. ${ }^{9,14,15}$

We present two brothers with CMTX and recurrent selflimited attacks of hemiplegia, dysarthria and ataxia. Differential 
diagnoses considered in our patients included infarction, infection, leukodystrophy, vasculitides and toxin ingestion. Although reversible posterior leukoencephalopathy may show symmetrical involvement of the occipital white matter as in Case 2 , our patient was normotensive and had not been exposed to any substances that can cause leukoencephalopathy.

Considering the brothers' transient clinical and MRI findings and subsequent development of classic migraines, initial consideration was given to a channelopathy - specifically the diagnosis of familial hemiplegic migraine (FHM) as $60 \%$ of patients with FHM also have symptoms of basilar insufficiency. However, the absence of characteristic headache in Case 1 at the time of initial presentation went against this diagnosis.

Given the unique MRI in Case 2, a literature search was performed looking specifically for the transient neurological syndrome in CMTX and abnormal MRI findings similar to our patient. Only eleven such cases excluding our own have been reported to date..$^{10-13,17}$ Most are male and have an uncharacteristically similar clinical and radiological profile seemingly triggered by respiratory infections, travel to a high altitude or trauma. These patients are genetically dissimilar but all have mutations in the $\mathrm{Cx} 32$ protein that would theoretically cause non functional gap junctions ${ }^{13}$ in the CNS.

A missense GTC-ATG mutation in the connexin 32 gene predicted to cause an amino acid variation V139 M was later found in both brothers and their mother. This mutation localises to the third transmembrane domain of the connexin32 protein. The third transmembrane domain is predicted to form the central pore of gap junctions. The V139M mutation has been described in CMTX patients with peripheral neuropathy ${ }^{16}$ but has never been reported with the transient CNS phenotype. Kleopa et al ${ }^{18}$ using transfection and cellular expression studies have shown that $\mathrm{Cx} 32$ mutants with third transmembrane domain mutations are retained within the Golgi apparatus and thus do not form functional gap junctions.

Why only a few patients with CMTX develop the above clinical CNS phenotype is unknown. At a cellular level, whether mutant $\mathrm{Cx} 32$ causes a dominant negative effect on other connexins expressed in oligodendrocytes or a direct toxic effect in oligodendrocytes is still unclear. ${ }^{13,18}$ We feel that dedicated reporting of various mutations associated with this phenotype will help further the genotype-phenotype correlation in CMTX.

\section{REFERENCES}

1. Klein CJ. The inherited neuropathies. Neurol Clin. 2007; 25(1):173207.

2. Nicholson G, Corbett A. Slowing of central conduction in X-linked charcot-marie-tooth neuropathy shown by brain stem auditory evoked responses. J Neurol Neurosurg Psychiatry. 1996; 61(1): 43-6.

3. Hisama FM, Lee HH, Vashlishan A, Tekumalla P, Russell DS, Auld E, et al. Clinical and molecular studies in a family with probable $\mathrm{X}$-linked dominant charcot-marie-tooth disease involving the central nervous system. Arch Neurol. 2001; 58(11):1891-6.

4. Marques W, Jr, Sweeney JG, Wood NW, Wroe SJ, Marques W. Central nervous system involvement in a novel connexin 32 mutation affecting identical twins. J Neurol Neurosurg Psychiatry. 1999; 66(6):803-4.

5. Karadima G, Panas M, Floroskufi P, Kalfakis N, Vassilopoulos D. A V38A mutation in X-linked charcot-marie-tooth neuropathy with unusual clinical features. J Neurol. 2004; 251(2):222-3.
6. Takashima H, Nakagawa M, Umehara F, Hirata K, Suehara M, Mayumi H, et al. Gap junction protein beta 1 (GJB1) mutations and central nervous system symptoms in X-linked charcotmarie-tooth disease. Acta Neurol Scand. 2003; 107(1):31-7.

7. Kassubek J, Bretschneider V, Sperfeld AD. Corticospinal tract MRI hyperintensity in X-linked charcot-marie-tooth disease. J Clin Neurosci. 2005; 12(5):588-9.

8. Murru MR, Vannelli A, Marrosu G, Cocco E, Corongiu D, Tranquilli S, et al. A novel $\mathrm{Cx} 32$ mutation causes X-linked charcot-marie-tooth disease with brainstem involvement and brain magnetic resonance spectroscopy abnormalities. Neurol Sci. 2006; 27(1):18-23.

9. Basri R, Yabe I, Soma H, Matsushima M, Tsuji S, Sasaki H. Xlinked charcot-marie-tooth disease (CMTX) in a severely affected female patient with scattered lesions in cerebral white matter. Intern Med. 2007; 46(13):1023-7.

10. Paulson HL, Garbern JY, Hoban TF, Krajewski KM, Lewis RA, Fischbeck KH, et al. Transient central nervous system white matter abnormality in X-linked charcot-marie-tooth disease. Ann Neurol. 2002; 52(4):429-34.

11. Schelhaas HJ, Van Engelen BG, Gabreels-Festen AA, Hageman G, Vliegen JH, Van Der Knaap MS, et al. Transient cerebral white matter lesions in a patient with connexin 32 missense mutation. Neurology. 2002; 24:59(12):2007-8.

12. Panas M, Kalfakis N, Karadimas C, Vassilopoulos D. Episodes of generalized weakness in two sibs with the $\mathrm{C} 164 \mathrm{~T}$ mutation of the connexin 32 gene. Neurology. 2001; 57(10):1906-8.

13. Taylor RA, Simon EM, Marks HG, Scherer SS. The CNS phenotype of X-linked charcot-marie-tooth disease: More than a peripheral problem. Neurology. 2003; 61(11):1475-8.

14. Isoardo G, Di Vito N, Nobile M, Benetton G, Fassio F. X-linked charcot-marie-tooth disease and progressive-relapsing central demyelinating disease. Neurology. 2005; 65(10):1672-3.

15. Parman Y, Ciftci F, Poyraz M, Halefoglu AM, Oge AE, Eraksoy M, et al. X-linked charcot-marie-tooth disease and multiple sclerosis. J Neurol. 2007; 254(7):953-5.

16. Bergoffen J, Scherer SS, Wang S, Scott MO, Bone LJ, Paul DL, et al. Connexin mutations in X-linked charcot-marie-tooth disease. Science. 1993; 262(5142):2039-42.

17. Hanemann CO, Bergmann C, Senderek J, Zerres K, Sperfeld AD. Transient, recurrent, white matter lesions in X-linked charcotmarie-tooth disease with novel connexin 32 mutation. Arch Neurol. 2003; 60(4):605-9.

18. Kleopa KA, Zamba-Papanicolaou E, Alevra X, Nicolaou P, Georgiou DM, Hadjisavvas A, et al. Phenotypiac and cellular expression of two novel connexin mutations causing CMT1X. Neurology. 2006; 66:396-402. 